Supplementary Material:

\title{
Physics-based computational protein design: an update
}

David Mignon, Karen Druart, Eleni Michael, Vaitea Opuu, Savvas Polydorides, Francesco Villa, Thomas Gaillard, Nicolas Panel, Georgios Archontis \& Thomas Simonson

\section{Computational methods}

Here we report details of the computational protocols used for the present applications.

\subsection{Whole protein design}

\subsubsection{Experimental amino acid frequencies}

We considered 3 protein domains from the PDZ family (Table S1). To define the target amino acid frequencies, we collected homologous sequences for each domain by searching the Non-redundant (NR) database with NCBI/Blast [1], Blosum62 scoring matrix, and the PDB sequence as query. We retained homologues with sequence identities vs. the query above $60 \%$. We used the HMMER algorithm [2] and the Superfamily tool [3] to identify and eliminate any Blast hits that did not belong to the same protein family as the query, leaving a total of 199 homologues. Finally, we aligned each query and its homologues with Clustal Omega [4].

Table S1: PDZ proteins targeted for redesign

\begin{tabular}{cccc}
\hline name & $\begin{array}{c}\text { PDB } \\
\text { code }\end{array}$ & residues & $\begin{array}{c}\text { number of } \\
\text { mutating positions }\end{array}$ \\
\hline NHREF & 1G9O & $9-99$ & 76 \\
Syntenin & 1R6J & $192-273$ & 72 \\
DLG2 & 2BYG & $186-282$ & 82 \\
\hline
\end{tabular}

\subsubsection{Structure preparation and energy matrices}

We began with the X-ray structures, listed in Table S1, and used the Modeler program to build any missing heavy atoms. We then placed hydrogens with the protX module of 
Proteus and did 1000 steps of conjugate gradient minimization with harmonic restraints of $3 \mathrm{kcal} / \mathrm{mol} / \AA^{2}$ on heavy atoms. With the resulting backbone structure and side chain rotamers, we calculated the interaction energy matrix (IEM) with protX as previously [5]. For GBLK, we used parameters optimized earlier [6]: a protein dielectric constant $\epsilon_{P}$ of $6.8, \lambda=5.33, S^{\text {alk }}=0.474, G_{\text {pol }}^{\text {ref }}=-0.838 \mathrm{kcal} / \mathrm{mol}, G_{\text {pol }}^{\text {free }}=-1.446 \mathrm{kcal} / \mathrm{mol}$. For GBSA, we used $\epsilon_{P}=8$ or 4 , and surface area parameters optmized earlier for CPD [7]. For the GB term, we used the Fluctuating Dielectric Boundary scheme (FDB) [8]. The IEM contains interactions between each residue and the backbone and between residue pairs (I, J), for all rotamers and for all side chain types except Gly and Pro. We used a slightly enhanced version of the Tuffery rotamer library [7, 9], along with "native" rotamers. Before the energy computation, for each state of residue I and residue pair I, $\mathrm{J}$ we performed 15 steps of minimization in order to alleviate bad steric contacts due to the rotamer approximation.

\subsection{3 $E_{u}$ optimization}

The unfolded state energies were optimized by likelihood maximization, as previously [5]. Parameters were optimized separately for each protein family. Briefly, separate parameters were assigned to buried and exposed positions in the folded structures of the proteins. Rounds of REMC were done, where each protein was simulated twice, with either half of its positions allowed to mutate. After each round, $\mathrm{MC}$ amino acid frequencies from a low temperature replica (thermal energy of $0.26 \mathrm{kcal} / \mathrm{mol}$ ) were compared to the experimental ones. Unfolded energies were updated with a linear update rule and a new round performed. To limit the number of adjustable parameters, early rounds grouped amino types into 11 classes, with shared parameters. In later rounds, each amino acid type had its own parameter.

\subsubsection{Sequence design and analysis}

Sequence design was performed using the optimized reference energy set obtained for each protein family. All positions (except Gly, Pro) could mutate freely into all types except Gly, Pro. We did REMC with eight replicas for 750 million MC steps. MC moves included rotamer changes and mutations at one or two positions [5]. Thermal energies ranged from 0.175 to $3 \mathrm{kcal} / \mathrm{mol}$. Periodic swaps were attempted between the conformations of two replicas $i, j$ (adjacent in temperature) every 7.5 million MC steps. The 10,000 lowest 
energy sequences sampled by any replica were kept for analysis. Amino acid frequencies from $\mathrm{MC}$ for the PDZ proteins are compared to the experimental ones in Table S2. The converged reference energies are also given. The values with GBSA are in Table S3.

The sequences were submitted to the Superfamily library of hidden Markov models [3], which attempts to classify sequences according to the SCOP structural classification of proteins [10]. We also compared the designed sequences to natural sequences from the Pfam the "RP55" alignment for each family, using the Blosum40 scoring matrix. We first aligned the designed sequences to the Pfam alignment for each family, using the MAFFT program [11] and the Blosum40 scoring matrix.

The diversity in the 10000 designed sequences from each PDZ domain was computed using the position-dependent sequence entropy [5]:

$$
S_{i}=-\sum_{t \in a a} f_{t}(i) \ln f_{t}(i)
$$

where $f_{t}(i)$ is the frequency of residue "type" $t$ at position $i$ of the designed sequences. Instead of the usual, 20 amino acid types, we employed six residue classes, corresponding to the following groups: $\{$ LVIMC $\},\{F Y W\},\{G\},\{\mathrm{ASTP}\},\{\mathrm{EDNQ}\}$, and $\{\mathrm{KRH}\}$. The entropies are exponentiated, then averaged over all positions. To obtain the diversity of all 30000 designed sequences from the three domains, we initially organized them into a multiple sequence alignment.

\subsection{Methionyl-tRNA synthetase design for $\beta$-Met activity}

The calculations followed closely the previous redesign of MetRS for $\alpha$-Met activity [12]. For the MC simulations, we used the GBLK solvent model, with the Fluctuating Dielectric Boundary (FDB) treatment $[8,12]$.

\subsection{Structural models}

A model of Escherichia coli (E. coli) MetRS with bound ATP was built using two crystal structures. First, we used the structure of $E$. coli MetRS bound to a methionyl adenylate (MetAMP) analog (PDB code 1PG0) [13], with the mobile KMSKS loop in its inactive, ATP-free conformation. Next, the ATP-bound loop conformation was modeled using the Leishmania major (L. major) MetRS complex with MetAMP and pyrophosphate (PDB code 3KFL) [14] by aligning common portions of the ligands in the two structures. 15 
Table S2: Amino acid composition of natural and designed PDZ sequences with GBLK

\begin{tabular}{|c|c|c|c|c|c|c|}
\hline \multirow[t]{2}{*}{ Residue } & \multicolumn{2}{|c|}{$\begin{array}{c}\text { Experimental } \\
\text { Frequencies }(\%)\end{array}$} & \multicolumn{2}{|c|}{$\begin{array}{c}\text { Proteus Frequencies } \\
(\%)\end{array}$} & \multicolumn{2}{|c|}{$\begin{array}{c}E_{t}^{\mathrm{uf}} \\
(\mathrm{kcal} / \mathrm{mol})\end{array}$} \\
\hline & Buried & Exposed & Buried & Exposed & Buried & Exposed \\
\hline ALA & 6.57 & 5.97 & 6.53 & 5.77 & 1.297 & 0.336 \\
\hline CYS & 1.26 & 0.54 & 1.23 & 0.73 & 1.970 & -0.534 \\
\hline THR & 4.70 & 6.37 & 4.70 & 6.06 & 1.158 & -2.320 \\
\hline GLU & 3.37 & 9.40 & 3.47 & 9.44 & -2.074 & -6.134 \\
\hline ASP & 3.98 & 6.65 & 3.89 & 6.67 & -3.233 & -7.435 \\
\hline PHE & 4.48 & 1.28 & 4.75 & 1.50 & 6.809 & 2.777 \\
\hline TRP & 0.01 & 0.01 & 0.31 & 0.33 & 8.133 & 4.434 \\
\hline ILE & 12.37 & 3.67 & 12.36 & 3.95 & 6.356 & 3.205 \\
\hline VAL & 16.47 & 4.90 & 16.25 & 4.96 & 2.415 & -1.108 \\
\hline LEU & 14.75 & 3.68 & 14.69 & 3.56 & 2.752 & -0.956 \\
\hline LYS & 6.26 & 8.10 & 6.11 & 8.51 & 1.709 & -1.800 \\
\hline MET & 1.93 & 0.77 & 2.20 & 0.90 & 1.661 & -1.652 \\
\hline ASN & 4.06 & 5.54 & 4.25 & 5.31 & 0.234 & -4.213 \\
\hline GLN & 2.04 & 4.67 & 2.16 & 4.21 & 1.491 & -2.683 \\
\hline SER & 3.51 & 6.58 & 3.65 & 6.49 & 1.055 & -1.565 \\
\hline $\mathrm{ARG}$ & 4.25 & 5.17 & 4.26 & 5.36 & -20.543 & -24.619 \\
\hline TYR & 2.08 & 1.02 & 1.85 & 0.93 & 3.779 & -0.384 \\
\hline HID & & & 1.85 & 0.95 & 20.964 & 17.240 \\
\hline HIE & 3.38 & 3.32 & 0.95 & 2.00 & 20.791 & 16.872 \\
\hline HIP & & & 0.01 & 0.00 & 21.971 & 18.037 \\
\hline PRO & 2.05 & 5.28 & 2.05 & 5.28 & - & - \\
\hline GLY & 2.49 & 17.08 & 2.49 & 17.08 & - & - \\
\hline Rmsd & & & $0.21 \%$ & $0.24 \%$ & & \\
\hline
\end{tabular}


Table S3: Amino acid composition of natural and designed PDZ sequences with $\operatorname{GBSA}\left(\epsilon_{P}=8\right)$

\begin{tabular}{|c|c|c|c|c|c|c|}
\hline \multirow[t]{2}{*}{ Residue } & \multicolumn{2}{|c|}{$\begin{array}{c}\text { Experimental } \\
\text { frequencies }(\%)\end{array}$} & \multicolumn{2}{|c|}{$\begin{array}{c}\text { Proteus frequencies } \\
(\%)\end{array}$} & \multicolumn{2}{|c|}{$\begin{array}{c}E_{t}^{\text {uf }} \\
(\mathrm{kcal} / \mathrm{mol})\end{array}$} \\
\hline & Buried & Exposed & Buried & Exposed & Buried & Exposed \\
\hline ALA & 6.57 & 5.97 & 6.95 & 7.37 & 0 & 0 \\
\hline CYS & 1.26 & 0.54 & 1.31 & 2.04 & -2.137 & -2.113 \\
\hline THR & 4.70 & 6.37 & 4.77 & 7.47 & -5.736 & -8.712 \\
\hline GLU & 3.37 & 9.40 & 3.65 & 10.64 & -15.956 & -20.69 \\
\hline ASP & 3.98 & 6.65 & 4.24 & 7.95 & -16.115 & 20.17 \\
\hline PHE & 4.48 & 1.28 & 4.45 & 2.54 & 3.142 & 1.865 \\
\hline TRP & 0.01 & 0.01 & 0.35 & 1.41 & 0.750 & -2.532 \\
\hline ILE & 12.37 & 3.67 & 12.05 & 3.01 & 4.622 & 1.472 \\
\hline VAL & 16.47 & 4.90 & 16.59 & 5.90 & 0.523 & -2.455 \\
\hline LEU & 14.75 & 3.68 & 15.16 & 5.05 & -0.243 & -4.277 \\
\hline LYS & 6.26 & 8.10 & 7.01 & 9.56 & -4.966 & -8.418 \\
\hline MET & 1.93 & 0.77 & 2.07 & 2.35 & -1.918 & -4.010 \\
\hline $\mathrm{ASN}$ & 4.06 & 5.54 & 4.44 & 6.94 & -15.654 & -20.31 \\
\hline GLN & 2.04 & 4.67 & 2.38 & 5.81 & -15.263 & -20.556 \\
\hline SER & 3.51 & 6.58 & 3.72 & 7.95 & -6.392 & -8.063 \\
\hline $\mathrm{ARG}$ & 4.25 & 5.17 & 4.76 & 6.72 & -45.249 & -49.887 \\
\hline TYR & 2.08 & 1.02 & 2.45 & 2.52 & -6.385 & -9.791 \\
\hline HID & & & 1.66 & 2.70 & 10.055 & 11.488 \\
\hline HIE & 3.38 & 3.32 & 1.96 & 0.20 & 9.887 & 5.551 \\
\hline HIP & & & 0.0 & 0.0 & 11.370 & 6.746 \\
\hline PRO & 2.05 & 5.28 & 2.05 & 5.28 & - & - \\
\hline GLY & 2.49 & 17.08 & 2.49 & 17.08 & - & - \\
\hline Rmsd & & & $0.23 \%$ & $0.87 \%$ & & \\
\hline
\end{tabular}


L. major loop residues were retained, and mutated into the E. coli types where different, using Scwrl4 ([15]. We then adjusted the geometry using 40 steps of conjugate gradient minimization to obtain the final model of E. coli MetRS with bound ATP and its KMSKS loop in the active, ATP-bound conformation. Adenylate and pyrophosphate present in $3 \mathrm{KFL}$ were used to position ATP in the binding site. $\mathrm{A} \mathrm{Mg}^{2+}$ ion coordinating the pyrophosphate in 3KFL was transferred to the present model. We used visual inspection to assign histidine protonation states. Other ionizable groups were in their standard protonation states. We refer to this model as MetRS:ATP.

We used a short MD simulation to adjust the protein backbone and the ligands further. Indeed, energy minimization alone seems to overspecialize the backbone for the wild type sequence. Protein was truncated $25 \AA$ from the ligand $\mathrm{P}_{\alpha}$, solvated in a large box of TIP3P water [16], then minimized for 100 conjugate gradient steps, with harmonic restraints applied to nonhydrogen atoms with force constants that decreased gradually over time from 5 to $0.5 \mathrm{kcal} / \mathrm{mol} / \AA^{2}$, except for groups near the truncation sphere, where the stronger restraints were kept. 575 ps of MD were done with NAMD [17].

Previously, we added $\alpha$-Met to the same MetRS:ATP complex to form MetRS:ATP: $\alpha$ Met [12]. Here, we added $\beta$-Met. We used the recent crystal structure (PDB code $6 \mathrm{SPN}$ ) of a complex between $E$. coli MetRS and $\beta$-Met [18]. We aligned that structure to MetRS:ATP to form MetRS:ATP: $\beta$-Met. The $6 \mathrm{SPN}$ crystal structure included two conformations for the carboxylate moiety of $\beta$-Met. One is much closer to the ATP, as required for catalysis, and we chose that geometry. The MetRS:ATP: $\beta$-Met was relaxed slightly by MD, as above.

To create the complex with the transition state for $\beta$-Met, we followed the same procedure as previously for $\alpha$-Met [12]. We started from MetRS:ATP: $\beta$-Met (above) and applied harmonic constraints to maintain carboxylate geometry. We added a harmonic bond energy term of length $2.4 \AA$ between the $\mathrm{P}_{\alpha}$ and the carboxylate carbon, and planar restraints for the resulting pentacoordinated $\alpha$ phosphate group. We used a few steps of minimization to reach the desired transition state geometry. We call this complex MetRS: $[\beta \text {-Met:ATP }]^{\ddagger}$. The $\alpha$-Met versionis called MetRS: $[\alpha \text {-Met:ATP }]^{\ddagger}$.

The ligand flexibility during MC (below) was limited to side chain rotamers for the Met moiety, taken from the Tuffery library [9]. The rest of the ligand was held fixed. 


\subsubsection{Energy matrix calculation}

The molecular mechanics force field was AMBER ff99SB for the protein [19, 20] and compatible parametrs were used for ATP [21]. For the $\beta$-Met backbone, we performed an $\mathrm{HF} / 6-31 \mathrm{G}^{*}$ ab initio calculation with Gaussian 9. Then, partial charges were chosen to reproduce the electrostatic potential [22]. This procedure is consistent with the rest of the Amber force field. Bonded and van der Waals parameters were assigned by analogy to the earlier $\alpha$-Met model and to the rest of the force field. For the transition state, we used the same procedure to obtain charges for the junction atoms between $\beta$-Met and ATP. Atomic charges are reported in Table S4.

An interaction energy matrix was calculated for each protein:ligand complex, using protX [23]. We followed the same protocol as in previous work [7, 12], where details are given. Briefly, the rotamer library was prepared from the 1995 library of Tuffery et al [9], the solvent model was of the GBLK form, with the Fluctuating Dielectric Boundary (FDB) treatment $[8,12]$, and with LK parameters reported earlier [6].

\subsection{Unfolded state model}

The unfolded state allows us to estimate roughly the stability change of predicted variants. We model the unfolded state as an extended peptide, whose energy is the sum of positiondependent terms [24]. For each mutating position, we computed the interaction energy between atoms in the side chain, the local backbone, and the two adjacent $\mathrm{C}_{\alpha}$ positions. The energy term for a given side chain type is then averaged over the best rotamers at each position. We call this energy $E_{u}(t)$, where $t$ is the side chain type. The resulting values are given in Table S5. Distinct values are used for the different His protonation states. Stability is computed here for the MetRS:ATP complex, meaning that ATP is in the binding site and the KMSKS loop is in its active conformation. The computed values therefore include a contribution due to ATP unbinding and loop rearrangement. Since the mutating residues are not in direct contact with ATP, we expect these contributions are rather independent of sequence.

\subsubsection{Sequence design}

We redesigned three amino acids close to the Met ligand side chain, as previously [12]: Leu13, Ala256, Ile297. Mutations into all types were allowed. The other positions kept their native types but could explore rotamers. Sequences and conformations were explored 
Table S4: Atomic charges for $\beta$-Met

\begin{tabular}{cccc}
\hline Atom & atom & & \\
name & type & $\beta$-Met:ATP & {$[\beta \text {-Met:ATP }]^{\ddagger}$} \\
\hline MG & MG & 1.5000 & 1.5000 \\
N & N3 & -0.3025 & -0.3025 \\
HN1 & H & 0.2770 & 0.2770 \\
HN2 & H & 0.2770 & 0.2770 \\
HN3 & H & 0.2770 & 0.2770 \\
CA & CT & -0.2298 & -0.2298 \\
HA & HP & 0.1208 & 0.1208 \\
CAA & CT & -0.0620 & -0.0620 \\
HAA2 & HC & -0.0554 & -0.0554 \\
HAA3 & HC & -0.0554 & -0.0554 \\
C & C & 0.8326 & 0.9610 \\
O & O & -0.7856 & -0.7856 \\
OX & OX & -0.7257 & -0.7517 \\
PB & P & 1.3586 & 1.4664 \\
O1B & O2 & -0.8280 & -0.9582 \\
O2B & O2 & -0.8933 & -0.8900 \\
O3B & OS & -0.5746 & -0.6252 \\
PA & PA & 1.2412 & 1.1805 \\
O1A & O2 & -0.6153 & -0.6138 \\
O2A & O2 & -0.7853 & -0.7016 \\
O3A & OS & -0.7561 & -0.8680 \\
O5 & OS & -0.5025 & -0.4478 \\
C5 & CT & 0.0558 & 0.0558 \\
\hline & & & \\
\hline
\end{tabular}

by $\mathrm{MC}$ with the protMC module of Proteus. Simulations were run for $10^{9}$ steps, with thermal energy $0.6 \mathrm{kcal} / \mathrm{mol}$ and move probabilities of $0.9,0.1,0.9$, and 0.1 , respectively for single rotamer, single mutation, pair of rotamers, and mutation-rotamer moves. An adaptive bias was optimized in certain simulations, with parameters reported earlier [12]. 
Table S5: Unfolded energies $E_{u}(\mathrm{kcal} / \mathrm{mol})$ for the MetRS design calculations

\begin{tabular}{lrlr}
\hline type & $E_{u}$ & type & $E_{u}$ \\
\hline ALA & 6.80 & ILE & 10.15 \\
ARG & -18.79 & LEU & 5.99 \\
ASN & 0.31 & LYS & 4.61 \\
ASP & -4.23 & MET & 5.74 \\
CYS & 5.91 & PHE & 9.50 \\
GLN & 2.58 & SER & 3.58 \\
GLU & -1.24 & THR & 3.07 \\
HIS $_{\delta}$ & 23.27 & TRP & 11.13 \\
HIS $_{\epsilon}$ & 22.50 & TYR & 6.21 \\
HIS $^{+}$ & 27.14 & VAL & 5.46 \\
\hline
\end{tabular}

\section{References}

[1] Schaffer, A. A., Aravind, L., Madden, T. L., Shavirin, J. L., Spouge, S., Wolf, Y. I., Koonin, E. V., And Altschul, S. F. Improving the accuracy of PSI-BLAST protein database searches with composition-based statistics and other refinements. Nucl. Acids Res. 29 (2001), 2994-3005.

[2] HMMER: biosequence analysis using profile Hidden Markov Models. available at http://hmmer.org.

[3] Wilson, D., Madera, M., Vogel, C., Chothia, C., and Gough, J. The SuPerFAMILY database in 2007: families and functions. Nucl. Acids Res. 35 (2007), D308$-\mathrm{D} 313$.

[4] Sievers, F., And Higgins, D. G. Clustal Omega for making accurate alignments of many protein sequences. Prot. Sci. 27 (2018), 135-145.

[5] Mignon, D., Panel, N., Chen, X., Fuentes, E. J., and Simonson, T. Computational design of the Tiam1 PDZ domain and its ligand binding. J. Chem. Theory Comput. 13 (2017), 2271-2289.

[6] Michael, E., Polydorides, S., Simonson, T., And Archontis, G. Simple models for nonpolar solvation: parametrization and testing. J. Comput. Chem. 38 (2017), 2509-2519. 
[7] Gaillard, T., And Simonson, T. Full protein sequence redesign with an mmgbsa energy function. J. Chem. Theory Comput. 13 (2017), 4932-4943.

[8] Villa, F., Mignon, D., Polydorides, S., and Simonson, T. Comparing pairwiseadditive and many-body generalized born models for acid/base calculations and protein design. J. Comput. Chem. 38 (2017), 2396-2410.

[9] Tuffery, P., Etchebest, C., Hazout, S., And Lavery, R. A new approach to the rapid determination of protein side chain conformations. J. Biomol. Struct. Dyn. 8 (1991), $1267-1289$.

[10] Andreeva, A., Howorth, D., Brenner, S. E., Hubbard, J. J., Chothia, C., And Murzin, A. G. SCOP database in 2004: refinements integrate structure and sequence family data. Nucl. Acids Res. 32 (2004), D226-229.

[11] Katoh, K., And Standley, D. M. MAFFT multiple sequence alignment software version 7: improvements in performance and usability. Molec. Biol. Evol. 30 (2013), 772-780.

[12] Opuu, V., Nigro, G., Gaillard, T., Mechulam, Y., Schmitt, E., and Simonson, T. Adaptive landscape flattening allows the design of both enzyme:substrate binding and catalytic power. PLoS Comp. Biol. 16 (2020), e1007600.

[13] Crépin, T., Schmitt, E., Mechulam, Y., Sampson, P. B., Vaughan, M. D., Honek, J. F., AND Blanquet, S. Use of analogues of methionine and methionyl adenylate to sample conformational changes during catalysis in Escherichia coli methionyl-tRNA synthetase. J. Mol. Biol. 332 (2003), 59-72.

[14] Larson, E. T., Kim, J. E., Zucker, F. H., Kelley, A., Mueller, N., Napuli, A. J., Verlinde, C. L., Fan, E., Buckner, F. S., Voorhis, W. C. V., Merritt, E. A., AND HoL, W. G. Structure of Leishmania major methionyl-tRNA synthetase in complex with intermediate products methionyl adenylate and pyrophosphate. Biochimie 93 (2011), $570-582$.

[15] Krivov, G. G., Shapalov, M. V., and Dunbrack, R. L. Improved prediction of protein side-chain conformations with SCWRL4. Proteins 77 (2009), 778-795.

[16] Jorgensen, W. L., Chandrasekar, J., Madura, J., Impey, R., And Klein, M. Comparison of simple potential functions for simulating liquid water. J. Chem. Phys. 79 (1983), 926-935. 
[17] Phillips, J. C., Braun, R., Wang, W., Gumbart, J., Tajkhorshid, E., Villa, E., Chipot, C., Skeel, R. D., Kale, L., And Schulten, K. Scalable molecular dynamics with NAMD. J. Comput. Chem. 26 (2005), 1781-1802.

[18] Nigro, G., Bourcier, S., Lazennec-Schurdevin, C., Schmitt, E., Marlière, P., , AND Mechulam, Y. Use of $\beta 3$-methionine as an amino acid substrate of Escherichia coli methionyl-tRNA synthetase. J. Struct. Biol. 209 (2020), 107435.

[19] Wang, L., Magliery, T., Liu, D. R., And Schultz, P. G. A new functional suppressor tRNA/aminoacyl-tRNA synthetase pair for the in vivo incorporation of unnatural amino acids into proteins. J. Am. Chem. Soc. 122 (2000), 5010-5011.

[20] Hornak, V., Abel, R., Okur, A., Strockbine, B., Roitberg, A., and SimmerLING, C. Comparison of multiple Amber force fields and development of improved protein backbone parameters. Proteins 65 (2006), 712-725.

[21] Meagher, K. L., Redman, L. T., and Carlson, H. A. Development of polyphosphate parameters for use with the AMBER force field. J. Comput. Chem. 24 (2003), 1016-1025.

[22] Cornell, W., Cieplak, P., Bayly, C., Gould, I., Merz, K., Ferguson, D., Spellmeyer, D., Fox, T., Caldwell, J., and Kollman, P. A second generation force field for the simulation of proteins, nucleic acids, and organic molecules. J. Am. Chem. Soc. 117 (1995), 5179-5197.

[23] Simonson, T. The Proteus software for computational protein design. https://proteus.polytechnique.fr, Ecole Polytechnique, Paris, 2019.

[24] Pokala, N., And Handel, T. M. Energy functions for protein design: adjustment with protein-protein complex affinities, models for the unfolded state, and negative design of solubility and specificity. J. Mol. Biol. 347 (2005), 203-227. 